\title{
A combined local facial feature extraction for age estimation
}

\author{
Xu Shasha, Zhang Hongbin, Wang Jianwen \\ Computer College Beijing University of Technology Beijing, 100124, China \\ shasha319@emails.bjut.edu.cn zhb@public.bta.net.cn wangjianwen24@126.com
}

\begin{abstract}
Age estimation is an important enabling capability for its potential applications in the near future, especially in supervision of minors, retrieving photograpgs at specific age ranges in ephotoalbums, and predicting future facial appearance of subjects . Due to the intrinsic and external factors the age estimation is a very complex task. One of the most important steps is feature extraction . However, Single feature extraction methods are commonly used, and all of them have their own drawbacks and limitations. In this paper, LBP combined with sp-PCA[1] method for age feature extraction is proposed. Addtionally in order to make the intra-class more compact, extra-class more loose, We adopt the Relevant Component Analysis(RCA) for distance metric adjustment .

Index Terms --sp-PCA , LBP , RCA (Relevant Component Analysis)
\end{abstract}

\section{Introduction}

Face images convey a significant amount of information including info about the identity, emotional state, ethnic origin, gender, age, and head orientation of a person shown in a face image. This type of information plays a significant role during face-to-face communication between humans. The use of facial information during interaction is made possible by the remarkable ability of humans to accurately recognize and

interpret faces and facial gestures. The real-time systems capable of identifying faces, recognizing emotions, gender and head orientation have been developed. Recently age estimation has attracted attention due to its wide applications, such as supervision of minors 、 demographic, commercial advertisement and so on.However the age estimation is a very complex problem for the intrinsic and external factors such as diet, environment, culture, social and gender factors.

In the rest of this paper,firstly we present our work in brief;Section 2 overview the previous work related to the study of the age classification and our main work ;in section 3 and section4,our methods used for facial feature extraction are discussed; then the experiment results are reported and analyzed; Finally, conclusion are drawn in section 5 .

\subsection{Related}

Age estimation for facial images is firstly peformed by Kwon and Lobo, they [2] classified ages from facial images into 3 age groups as babies, young adults and senior adults. They used cranio-facial development theory and skin wrinkle analysis in their study.

Lanitis et.al in [3] mainly investigated 3 different classifiers for automatic age estimation. They compared a quadratic function based classifier, a shortest distance classifiers and neural network based classifiers. Supervised and unsupervised neural networks are investigated for age estimation experiments.

Geng et.al[4] proposed a subspace approach named AGES (Aging pattern Subspace) for automatic age estimation by defined an image sequence of one subject as an aging pattern,which is represented using PCA model , and age estimation is performed by searching the proper position at age patterns. Although AGES got great success in age estimation, it heavily depend on age distribution of the training set, if there is no training sample at one specific age, it is impossible to correctly predict the testing instance of the corrspongding age .

Lanitis [5] performed automatic age estimation experiments using four different facial regions: the whole face (including the hairline), the whole internal face, the upper part of the face and the lower parts of the face. According to his result, the internal part of the face and particularly the area around the eyes proved to be the most significant for the task of automatic age estimation.

Shenglan Ben[6] suggested that different facial regions contain different individual information, and the aging effect is various in different facial regions; facial organs would mature at different ages, and their significant age related variation would appear at different ages. They claimed that using selective region ensemble can improve age estimation performance, and age range-based selective region ensemble is even superior to the global ensemble .

Fatemeh[6] respectively used subpatternd-based LBP , spPCA and mPCA approaches for the age classification, The emperiments are performed using three different datasets. The result show the efficiency of LBP method over the other methods. Above all, they firstly used spPCA and mPCA for age estimation.

Wei [7]proposed a new estimation approach considering the intrinsic factor of human age ,after face extractor ,RCA is utilized to achieved a suited space for neighbor searching ; then LLPP and MFA are respectively trained to reduced the feature dimensions ; almost in every step ,they considered the label of the images .

In all, many researchers have claimed as face age characteristics mainly displays in the texture characteristics, local features are widely used in their facial age estimation, and some of them have considered different facial parts differently effect the age estimation, and also some used the label of the subject for distance metric adjustment, but few of 
them considered using combined method. Each feature representation has its advantage and disadvantage .Hence , combined local facial features extraction for age estimation are investigated in this study.

\subsection{Contribution of Our Work}

Face age characteristics mainly displays in the texture characteristics, LBP is the most popular facial age model used to project such as in[8-11], it has been proved a really powerful messure of image texture with excellent result in terms of accuracy and computational complexity in many empirical studies. As the dimension of LBP features is usually too high to train a robust age classifier, PCA is used to reduce the dimension of correlated coefficients in an optimal way. However, the usage of the PCA also makes features uncorrelated. What is more, the significance of different facial parts in the task of the age estimation are vared $[5,6,12]$, the traditionarily using of PCA may lead to vital feature lost . In this paper, LBP combined with sub- PCA method age feature extraction is proposed,

Addtionally, considering the label, in order to make the intraclass more compact, extra-class more loose, we adopt the Relevant Component Analysis for distance metric adjustment . This work investigates different methods on single and fused facial feature representation. We performed the experiments on FG-Net facial age database. Our system is showed as the figure 1.

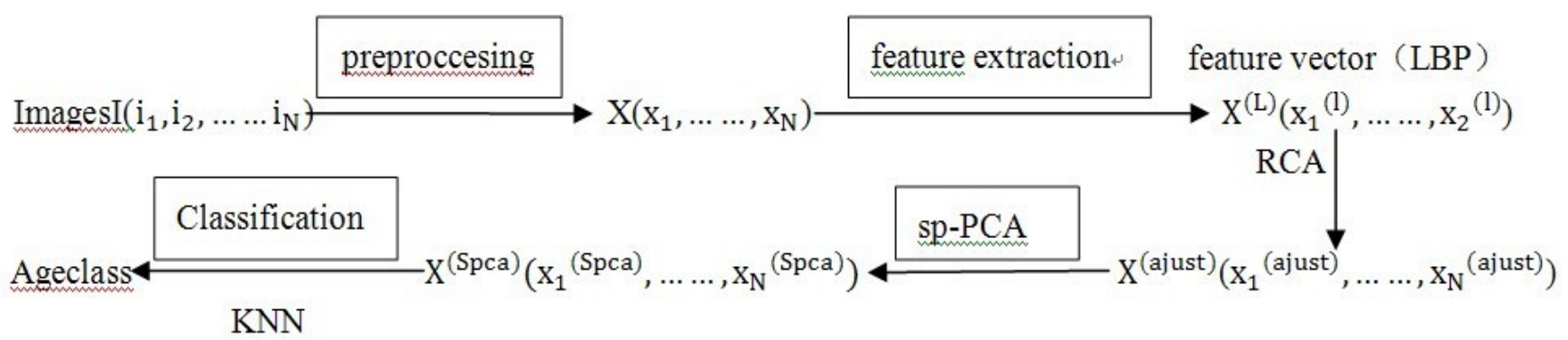

Figure1. the flowchart of our system

\subsection{Face Pre-processing:}

Face pre-processing is an important step of the facial image age estimation, As the images in the FG-Net database differ from each other in size, background, illumination ,pose and so on.Above all, when using LBP and sp-PCA to extract the features, there are strict requirements for its placement, processing reliability has an important influence on the overall result . Firstly, the facial image are rotated to make the eyes in a horizontal line, and then cropping is implemented manually to make the images in the same size.To eliminate the light effects, histogram equalization is finally utilized. In the figure2, the upper are the original, the under are the processed.

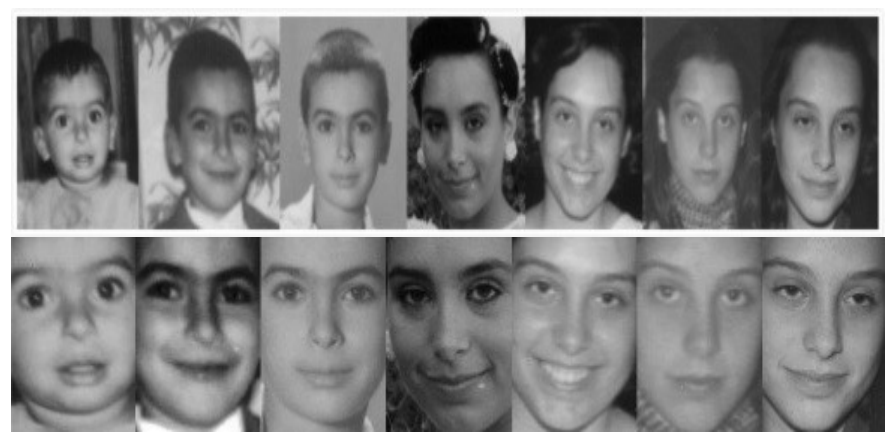

Figure2.original and processed images

\subsection{Feature Extraction:}

The images are patitioned into non-overlapping equallysize blocks, LBP texture descriptors are extracted from each region independently. The descriptors are then concatenated to form a global description of the face. In order to build local descriptions, LBP extract the features from the image by assigning the labels into the pixels and thresholding pixels in a certain neighborhood with the center value. LBP constructs the code for every pixel and considers the result as a binary number. The occurrence histogram of these labels is used as texture features or descriptors. Finally different histograms can be combined to form a code image. The number of the training subjects is N, An image is divided into m blocks.

$$
\mathrm{X}=\left\{\mathrm{x}_{\mathrm{n}} \in \mathrm{R}^{\mathrm{d}}\right\}_{\mathrm{n}=1}^{\mathrm{N}} ; \mathrm{x}_{\mathrm{n}}=\left\{\mathrm{x}_{\mathrm{n} 1}, \ldots \ldots \mathrm{x}_{\mathrm{nm}}\right\},
$$

for every block $\mathrm{x}_{\mathrm{nm}}$ LBP texture descriptors are extracted from each region independently as $\mathrm{x}_{\mathrm{nm}}^{(\mathrm{L})}$,

$$
\begin{aligned}
& \mathrm{x}_{\mathrm{n}}^{(\mathrm{L})}=\left\{\mathrm{x}_{\mathrm{n} 1}^{(\mathrm{L})}, \mathrm{x}_{\mathrm{n} 2}^{(\mathrm{L})}, \ldots \ldots \mathrm{x}_{\mathrm{nm}}^{(\mathrm{L})}\right\} ; \\
& \mathrm{x}^{(\mathrm{L})}=\left(\mathrm{x}_{1}{ }^{(\mathrm{L})}, \mathrm{x}_{2}{ }^{(\mathrm{L})}, \ldots \ldots \mathrm{x}_{\mathrm{n}}{ }^{(\mathrm{L})}\right)^{\mathrm{T}} ;
\end{aligned}
$$

As we all know, PCA is an unsupervised dimensionality reduction method, it just reduce the dimension of correlated coefficients in an optimal way, not considering the class. in order to make the intra-class more compact, extra-class more loose, We adopt the Relevant Component Analysis for distance metric adjustment. 


\section{Table1.The algorithm of RCA}

Preseting :

$\mathrm{x}^{\mathrm{L}}=\left\{\mathrm{x}_{\mathrm{n}}^{(\mathrm{L})} \in \mathrm{R}^{\mathrm{d}}\right\}_{\mathrm{n}=1}^{\mathrm{N}} ; \mathrm{C}=\left\{\mathrm{c}_{\mathrm{n}} \in \mathrm{C}\right\}_{\mathrm{n}=1}^{\mathrm{N}} ; \mathrm{X}_{\mathrm{i}}^{(\mathrm{L})}$ as the feature set containing all feature samples with label $\mathrm{C}_{\mathrm{i}}$, the number of the samples in $\mathrm{X}_{\mathrm{i}}^{(\mathrm{L})}$ is denoted as $\mathrm{N}_{\mathrm{i}}$;

(1) For each class $C_{i}$,compute the mean vector

$$
\mathrm{u}_{\mathrm{i}}=\frac{1}{\mathrm{~N}_{\mathrm{i}}} \sum_{\mathrm{x}_{\mathrm{n}}^{(\mathrm{L})}} \in_{\mathrm{X}_{\mathrm{i}}^{(\mathrm{L})}} \mathrm{X}_{\mathrm{n}}^{(\mathrm{L})}
$$

(2) $\left.s_{i}=\frac{1}{N_{i}} \sum_{x_{n}^{(L)} \in_{X}^{(L)}}^{(X} \underset{n}{(L)}-u_{i}\right)\left(X_{n}^{(L)}-u_{i}\right)^{T}$;

(3) $S=\frac{1}{N} \sum_{i=1}^{c} N_{i} \times s_{i}$ gain the choosed characteristic vector matrix $\mathrm{W}$

(4) $\mathrm{X}^{\text {(ajust) }}=\mathrm{x}^{(\mathrm{L})} \times \mathrm{w}^{\mathrm{T}}$

After the adjustment, as the significance of different facial parts in the task of the age estimation are vared, and PCA only reduce the dimension of correlated coefficients between the features, the traditionarily using of PCA may lead to vital feature lost. sp-PCA is applied on individual subpatterns sets and find the corresponding projection subvetors, that is to say, as the an image is patitioned into nonoverlapping equally size subpatterns, PCA is performed on every subpattern, the corresponding sub-features are obtained, and then all the sub-features are concatenated to form a global feature of the original whole pattern.

\section{Table2.The algorithm of sp-PCA}

\section{Presetting:}

(1) after processed with RCA, each subject could also be

$$
\text { expressed as } X_{n}^{\text {(ajust) }}=\left\{x_{n 1}^{\text {(ajust) }}, \mathrm{x}_{\mathrm{n} 2}^{\text {(ajust) }}, \ldots \ldots \mathrm{x}_{\mathrm{nm}}^{\text {(ajust) }}\right\}
$$

(2) the global features

$$
\mathrm{X}^{\text {(ajust) }}=\left(\mathrm{x}_{1}{ }^{\text {(ajust) }}, \mathrm{x}_{2}{ }^{\text {(ajust) }}, \ldots \ldots \mathrm{x}_{\mathrm{n}}{ }^{\text {(ajust) }}\right)^{\mathrm{T}} \text {; }
$$

(3) the blocks $\mathrm{S}_{\mathrm{i}}^{\text {Spca }}=\left(\mathrm{x}_{1 \mathrm{i}}^{\text {(ajust) }}, \mathrm{x}_{2 \mathrm{i}}^{\text {(ajust) }}, \ldots \ldots, \mathrm{x}_{\mathrm{Ni}}^{\text {(ajust) }}\right)$;

Algorithm:

(1) For each subpattern $S_{i}^{\text {Spca }}$, gain the PCA feature, choose the principal component as $\mathrm{PCA}_{\mathrm{i}}=\operatorname{eigs}\left(\mathrm{S}_{\mathrm{i}}^{\mathrm{Spca}}\right)$

(2) combined all the characteristic vector matrixs,to gain the global characteristic vector matrix $\mathrm{PCA}=\left\{\mathrm{PCA}_{1}, \mathrm{PCA}_{2}, \ldots . \mathrm{PCA}_{\mathrm{m}}\right\}$

(3) the final feature $\mathrm{X}_{\mathrm{i}}^{\text {(Spca) }}=\mathrm{x}_{\mathrm{i}}^{\text {(ajust) }} \times \mathrm{PCA}_{\mathrm{i}}$

\subsection{Classification:}

In this paper, finally,in the classification phase, we used KNN (k-nearest neighbor) classifier in the age estimation for the following two reasons : firstly ,KNN classifier is fast and easy to implement ; the second and the most important is to remove the classifier factor and prove our proposed feature extraction method is better than [10], which used KNN classifier in the classification phase.

\section{Results}

The experiments are conducted using the FG-Net aging database, FG-Net aging database contain 1002 images from 82 individuals at different ages.In this study, we used frontal images with free of glass, beard and moustache. We divided ages into five age groups with ten years intervals as: $0 \sim 10$, 11 20, 21 30, 31 40,41 60. The facial images are divided into $25,35,49,63$, and 81 subpatterns. The results are presented in table 3 .

Experiment results show that $\mathrm{LBP}+\mathrm{sp}-\mathrm{PCA}$ is better than both LBP and sp-PCA, RCA adjustment is necessary, and the number of the su-bpattern effect the result.The best result is $\mathrm{LBP}+$ sp-PCA with $63(7 \times 9)$ blocks.

Table 3. age classification rates

\begin{tabular}{|c|c|c|c|c|}
\hline Range & LBP & sp-PCA & LBP+sp-PCA & LBP+sp-PCA+RCA \\
\hline $0 \sim 9$ & $74.7 \%$ & $71.3 \%$ & $76.3 \%$ & $79.3 \%$ \\
\hline $10 \sim 19$ & $73.3 \%$ & $71.6 \%$ & $75.7 \%$ & $77.1 \%$ \\
\hline $20 \sim 29$ & $70.3 \%$ & $67.4 \%$ & $72.6 \%$ & $74.3 \%$ \\
\hline $30 \sim 39$ & $68.9 \%$ & $59.3 \%$ & $73.3 \%$ & $76.7 \%$ \\
\hline $40 \sim 59$ & $78.3 \%$ & $69.1 \%$ & $79.4 \%$ & $81.2 \%$ \\
\hline
\end{tabular}

\section{Conclusion}

In this paper, LBP combined with sp-PCA method age feature extraction is proposed, and as the experiment showed it is better than the LBP and sp-PCA, RCA is utilized for distance metric adjustment . Compared with thestate-of-art algorithms the experiments demonstrate our approach achieves excellent performance.

\section{References:}

[1] Chen, S. and Y. Zhu, Subpattern-based principle component analysis. Pattern Recognition, 2004. 37(5): p. 1081-1083.

[2] Kwon, Y.H. and N.D.V. Lobo. Age classification from facial images. in Proceedings of the 1994 IEEE Computer Society Conference on Computer Vision and Pattern Recognition, June 21, 1994 - June 23, 1994. Seattle, WA, USA: Publ by IEEE.

[3] Lanitis, A., C. Draganova and C. Christodoulou, Comparing Different Classifiers for Automatic Age Estimation. IEEE Transactions on Systems, Man, and Cybernetics, Part B: Cybernetics, 2004. 34(1): p. 621-628.

[4] Geng, X., Z. Zhou and K. Smith-Miles, Automatic age estimation based on facial aging patterns. IEEE Transactions on Pattern Analysis and Machine Intelligence, 2007. 29(12): p. 2234-2240.

[5] Lanitis, A., On the significance of different facial parts for automatic age estimation. DSP 2002: 14th International Conference On Digital Signal Processing Proceedings, Vols 1 And 2.

[6] Ben, S., G. Su and Y. Wu. A two-step selective region ensemble for facial age estimation. in 4th International Conference on Intelligent Computing, ICIC 2008, September 15, 2008 - September 18, 2008. Shanghai, China: Springer Verlag.

[7] Chao, W.L., J.Z. Liu and J.J. Ding, Facial age estimation based on labelsensitive learning and age-oriented regression. Pattern Recognition, 2013. 46(3): p. 628-641.

[8] Txia, J. and C. Huang. Age estimation using AAM and local facial features. in IIH-MSP 2009 - 2009 5th International Conference on Intelligent Information Hiding and Multimedia Signal Processing, 
September 12, 2009 - September 14, 2009. Kyoto, Japan: IEEE Computer Society.

[9] Gunay, A. and V.V. Nablyev. Automatic age classification with LBP. in 2008 23rd International Symposium on Computer and Information Sciences, ISCIS 2008, October 27, 2008 - October 29, 2008. Istanbul, Turkey: Inst. of Elec. and Elec. Eng. Computer Society.

[10] Mirzaei, F. and O. Toygar. Facial age classification using subpatternbased approaches. in 2011 International Conference on Image
Processing, Computer Vision, and Pattern Recognition, IPCV 2011, July 18, 2011 - July 21, 2011. Las Vegas, NV, United states: CSREA Press.

[11] Sun, H.L., J. Shen and B. Chen, LBP Based Fast Face Recognition System on Symbian Platform. AASRI Conference On Computational Intelligence And Bioinformatics, 2012. 1: P. 276-281.

[12] Wang, H.L., et al. Effects of facial alignment for age estimation. in 11th International Conference on Control, Automation, Robotics and Vision, ICARCV 2010, December 7, 2010 - December 10, 2010. Singapore, Singapore: IEEE Computer Society. 